\title{
A CORRECTION OF SOME THEOREMS ON PARTITIONS
}

\author{
BY \\ PETER HAGIS, JR.
}

Theorem 4 in [1] gives a convergent series representation for $p_{a}(n)$, the number of partitions of a positive integer $n$ into positive summands of the form $m p \pm a_{j}$. Here $p$ is an odd prime and $a_{j}$ is an element of a set $a$ consisting of $r$ positive residues of $p$ each of which is less than $p / 2$. It is stated that the theorem holds for $n \geqq A / 12 p$, where $A=r p^{2}-6 \sum_{j=1}^{r} a_{j}\left(p-a_{j}\right)$. In the proof of this theorem the estimate $O\left(n^{1 / 3} k^{2 / 3+\varepsilon}\right)$ is used for certain complicated exponential sums. The proof of this estimate given in Theorem 2 of [1] depends on the fact that $(A-12 p n, k)=O(n)$. This is clearly false (in general) if $A=12 p n$ since $(0, k)=k$. Thus, Theorem 4 of [1] has been established only if $n>A / 12 p$.

Similar remarks apply to Theorem 6 in [2] in which a convergent series is obtained for $q_{a}(n)$, the number of partitions of $n$ into distinct positive summands of the form $m p \pm a_{j}$. Here it is asserted that the theorem holds for $n \geqq-A / 12 p$. However, the proof given is valid only if $n>-A / 12 p$. For the argument used to establish the required estimate $O\left(n^{1 / 3} k^{2 / 3+\varepsilon}\right)$ for the exponential sums involved does not hold if $A=-12 n p$. Thus, until (if ever) the necessary estimates contained in Theorems 2 and 3 of [1] and Theorems 2 through 5 of [2] can be justified for $n= \pm A / 12 p$ we must exclude these values of $n$ from consideration.

We conclude by giving a simple necessary condition for $A= \pm 12 p n$. From the definition of $A$ given above and the fact that either $a_{j}$ or $p-a_{j}$ is even we see that if $A= \pm 12 p n$ then $12 \mid r$.

\section{REFERENCES}

1. P. Hagis, Jr., A problem on partitions with a prime modulus $p \geqq 3$, Trans. Amer. Math. Soc. 102 (1962), 30-62.

2. - On a class of partitions with distinct summands, Trans. Amer. Math. Soc. 112 (1964), 401-415.

TEMPLE UNIVERSITY,

Philadelphia, Pennsylvania

Received by the editors September 21, 1964. 